PROCEEDINGS OF THE

AMERICAN MATHEMATICAL SOCIETY

Volume 33, Number 2. June 1972

\title{
COMPACT MULTILINEAR TRANSFORMATIONS
}

\author{
NISHAN KRIKORIAN
}

\begin{abstract}
It is well known that if a $C^{1}$ map between Banach spaces is compact, then its derivative is a compact operator. If the map is $C^{r}$, then what can be said about the compactness of its higher derivatives? This question leads us to a study of compact multilinear operators with the main result being that the higher derivatives of a compact map are such operators.
\end{abstract}

Let $E_{1}, \cdots, E_{m}, F$ be Banach spaces, and $T: E_{1} \times \cdots \times E_{m} \rightarrow F$ be a continuous $m$-multilinear transformation. We call $T$ jointly compact if it takes bounded sets into relatively compact sets, and we call $T$ separately compact if for each $j$ and any choice of points $e_{i} \in E_{i}, i \neq j$, the transformation $T\left(e_{1}, \cdots, e_{j-1}, e_{j+1}, \cdots, e_{m}\right): E_{j} \rightarrow F$ is compact. The object of this paper is to expose several simple properties of such transformations. We give an example $(\S \mathrm{A})$ where separate compactness does not imply joint compactness, and we show that the first $r$ derivatives of a compact $C^{r}$ function are jointly compact $(\S \mathrm{B})$. We then look at linear spaces of jointly compact transformations $(\S C)$ and investigate their behavior with respect to tensor products $(\S \mathrm{D})$.

(A) Let $T: l_{2} \times l_{2} \rightarrow l_{2}$ be defined as $T(a, b)=\left(a_{1} b_{1}, a_{2} b_{2}, \cdots\right)$ where $a=$ $\left(a_{1}, a_{2}, \cdots\right)$ and $b=\left(b_{1}, b_{2}, \cdots\right)$ are in $l_{2}$. Then $T$ is bilinear, continuous, and separately compact but not jointly compact.

Continuity follows from the inequalities

$$
\begin{aligned}
\|T(a, b)\| & =\left(\sum a_{i}^{2} b_{i}^{2}\right)^{1 / 2} \leqq\left(\sum a_{i}^{4}\right)^{1 / 4}\left(\sum b_{i}^{4}\right)^{1 / 4} \\
& \leqq\left(\sum a_{i}^{2}\right)^{1 / 4}\left(\sum b_{i}^{2}\right)^{1 / 4} \leqq 1 \text { for } \sum a_{i}^{2}, \sum b_{i}^{2} \leqq 1 .
\end{aligned}
$$

To show separate compactness fix $b \in l_{2}$ and take $N$ so that $\sum_{N^{+}+1}^{\infty} b_{i}^{2}<\varepsilon$. If $\|a\| \leqq 1$, then

$$
\begin{aligned}
T(a, b)= & \left(a_{1} b_{1}, \cdots, a_{N} b_{N}, 0, \cdots\right)+\left(0, \cdots, 0, a_{N+1} b_{N+1}, \cdots\right) \\
\subset & (\text { a closed } N \text {-dimensional ball of radius }\|b\|) \\
& +(\text { a set of radius }<\varepsilon) .
\end{aligned}
$$

Received by the editors September 12, 1971.

AMS 1970 subject classifications. Primary 46G05.

Key words and phrases. Multilinear transformation, compact map, $C^{r}$ map, Taylor formula, polarization identity, tensor product, projection norm, convex balanced hull.

(c) American Mathematical Society 1972 
Therefore, the set $\{T(a, b):\|a\| \leqq 1\}$ has a finite $\varepsilon$-net for any $\varepsilon>0$. On the other hand let $c^{n}=(0, \cdots, 0,1,0, \cdots)$ where 1 appears only in the $n$th slot. Then $T\left(c^{n}, c^{n}\right)=c^{n}$ has no convergent subsequences.

(B) Let $f$ be a $C^{r}$ differentiable map of an open set $U$ of a Banach space $E$ into a Banach space $F$ which takes bounded sets into relatively compact sets, then the derivatives $\left(D^{n} f\right)_{x}: E \times \cdots \times E \rightarrow F$ are jointly compact transformations for each $x \in U(1 \leqq n \leqq r)$.

The case $n=1$ appears in [4]. It is sufficient to show that the map $\phi: E \rightarrow F$ defined by $\phi(e)=\left(D^{n} f\right)_{x}(e, \cdots, e)$ is compact since by a polarization identity for symmetric transformations [2] we have

$$
\left(D^{n} f\right)_{x}\left(e_{1}, \cdots, e_{n}\right)=\sum_{\varepsilon_{1}, \cdots, \varepsilon_{n}=0}^{1}(-1)^{n-\left(\varepsilon_{1}+\cdots+\varepsilon_{n}\right)} \phi\left(\varepsilon_{1} e_{1}+\cdots+\varepsilon_{n} e_{n}\right) .
$$

Suppose $\phi$ is not compact. Then there is a sequence $\left\{e^{N}\right\}$ in the unit ball of $E$ such that

$\left\|\left(D^{n} f\right)_{x}\left(e^{N}, \cdots, e^{N}\right)-\left(D^{n} f\right)_{x}\left(e^{M}, \cdots, e^{M}\right)\right\| \geqq(n+2) \varepsilon$ for all $N \neq M$.

Let

$$
\begin{aligned}
w(x, e) \equiv & f(x+e)-f(x)-(D f)_{x}(e) \\
& -\frac{1}{2 !}\left(D^{2} f\right)_{x}(e, e)-\cdots-\frac{1}{n !}\left(D^{n} f\right)_{x}(e, \cdots, e)
\end{aligned}
$$

then by Taylor's formula [1] there is a $\delta$ such that $\|w(x, e)\|<\varepsilon\|e\|^{n}$ for all $\|e\|<\delta$. We now have

$$
\begin{aligned}
& \left\|f\left(x+\delta e^{N}\right)-f\left(x+\delta e^{M}\right)\right\| \\
& =\| \sum_{i=1}^{n} \frac{1}{i !}\left(D^{i} f\right)_{x}\left(\delta e^{N}, \cdots, \delta e^{N}\right) \\
& \quad-\sum_{i=1}^{n} \frac{1}{i !}\left(D^{i} f\right)_{x}\left(\delta e^{M}, \cdots, \delta e^{M I}\right)+w\left(x, \delta e^{N}\right)-w\left(x, \delta e^{M}\right) \| \\
& \geqq\left\|\left(D^{n} f\right)_{x}\left(\delta e^{N}, \cdots, \delta e^{N}\right)-\left(D^{n} f\right)_{x}\left(\delta e^{M}, \cdots, \delta e^{M I}\right)\right\| \\
& \quad-\sum_{i=1}^{n-1} \frac{1}{i !}\left\|\left(D^{i} f\right)_{x}\left(\delta e^{N}, \cdots, \delta e^{N}\right)-\left(D^{i} f\right)_{x}\left(\delta e^{M}, \cdots, \delta e^{M}\right)\right\| \\
& \quad-\left\|w\left(x, \delta e^{N}\right)\right\|-\left\|w\left(x, \delta e^{M}\right)\right\| \\
& \geqq(n+2) \delta^{n} \varepsilon-\sum_{i=1}^{n-1} \frac{\delta^{i}}{i !} \|\left(D^{i} f\right)_{x}\left(e^{N}, \cdots, e^{N}\right) \\
& \quad-\left(D^{i} f\right)_{x}\left(e^{M I}, \cdots, e^{M}\right) \|-\delta^{n} \varepsilon-\delta^{n} \varepsilon .
\end{aligned}
$$


Utilizing the compactness of $\left(D^{i} f\right)_{x}(1 \leqq i \leqq n-1)$ we pick a subsequence of $\left\{e^{N}\right\}$ denoted by $\left\{\bar{e}^{N}\right\}$ such that $\left\{\left(D^{i} f\right)_{x}\left(\bar{e}^{N}, \cdots, \bar{e}^{N}\right)\right\}$ are Cauchy sequences in $N$ for each $i$. We can therefore find a $P$ such that

$$
\left\|\left(D^{i} f\right)_{x}\left(\bar{e}^{N}, \cdots, \bar{e}^{N}\right)-\left(D^{i} f\right)_{x}\left(\bar{e}^{M I}, \cdots, \bar{e}^{M}\right)\right\| \leqq i ! \delta^{n-i} \varepsilon
$$

for $M, N \geqq P$ and $1 \leqq i \leqq n-1$. From the inequality above we obtain $\left\|f\left(x+\delta \bar{e}^{-N}\right)-f\left(x+\delta \bar{e}^{M}\right)\right\| \geqq(n+2) \delta^{n} \varepsilon-(n-1) \delta^{n} \varepsilon-\delta^{n} \varepsilon-\delta^{n} \varepsilon=\delta^{n} \varepsilon$ for $M, N \geqq P$. But this together with the fact that $\left\{x+\delta \bar{e}^{N}\right\}_{N=P}^{\infty}$ is a bounded sequence contradicts the compactness of $f$.

(C) Let $L\left(E_{1}, \cdots, E_{m} ; F\right)$ be the Banach space of continuous $m$-multilinear transformations from $E_{1} \times \cdots \times E_{m}$ to $F$, and let $C L\left(E_{1}, \cdots, E_{m} ; F\right)$ be the closed subspace of jointly compact maps. It is easy to see that the canonical Banach space isomorphism $L\left(E_{1}, L\left(E_{2}, \cdots, L\left(E_{m} ; F\right) \cdots\right)\right) \rightarrow$ $L\left(E_{1}, \cdots, E_{m} ; F\right)$ induces the injection

$$
C L\left(E_{1}, C L\left(E_{2}, \cdots, C L\left(E_{m} ; F\right) \cdots\right)\right) \rightarrow C L\left(E_{1}, \cdots, E_{m} ; F\right),
$$

however, this map is not necessarily surjective.

We let $H$ be any real Hilbert space and show that the inclusion $C L(H, C L(H ; R)) \subset C L(H, H ; R)$ is proper. Define the bilinear map $T(a, b)=(a, b)$ where $($,$) is the inner product in l_{2}$. Since $|(a, b)| \leqq$ $\|a\|\|b\|$, we clearly have $T \in C L(H, H ; R)$. But the map $a \rightarrow(a$, ) is not in $C L(H, C L(H ; R))$ since it gives an isomorphism of $H$ with its dual $H^{*}=C L(H ; R)$ and, therefore, cannot be compact.

(D) If $E, F, G$ are Banach spaces and $E \hat{\otimes} F$ is the completion of $E \otimes F$ under the projective norm [3], then the canonical (algebraic) isomorphism $L(E, F ; G) \rightarrow L(E \hat{\otimes} F ; G)$ induces the isomorphism

$$
C L(E, F ; G) \rightarrow C L(E \hat{\otimes} F ; G) .
$$

Given any continuous bilinear $T$ there is a continuous linear $\hat{T}$ such that the diagram

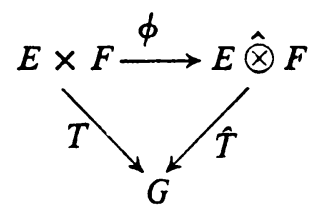

commutes; $\phi$ is the obvious canonical map. The isomorphism above it given by the correspondence between $T$ and $\hat{T}$. Suppose $\hat{T}$ is compact. Since $\|e \otimes f\|=\|e\|\|f\|$, we have that $\phi$ takes bounded sets to bounded 
sets which under $\hat{T}$ go into relatively compact sets. Therefore, $\hat{T} \cdot \phi=T$ is compact.

Conversely assume $T$ is compact. Every element $\omega$ of the open unit ball of $E \times F$ has a representation $\omega=\sum_{n=0}^{\infty} \lambda_{n}\left(e_{n} \otimes f_{n}\right)$ where $\left\{e_{n}\right\}$ and $\left\{f_{n}\right\} \rightarrow 0$ in the open unit balls $U$ and $V$ of $E$ and $F$ respectively and $\sum_{n=0}^{\infty}\left|\lambda_{n}\right|<1$ [3]. Therefore the closed ball of radius $\frac{1}{2}$ in $E \hat{\otimes} F$ is contained in the closure (cl) of the convex balanced hull (cbh) of $\phi(U \times V)$. Applying $\hat{T}$ we get

$$
\begin{aligned}
\hat{T}(\omega) & \in \hat{T}(\operatorname{cl}(\operatorname{cbh}(\phi(U \times V)))) \subset \operatorname{cl}(\hat{T}(\operatorname{cbh}(\phi(U \times V)))) \\
& =\operatorname{cl}(\operatorname{cbh}(\hat{T}(\phi(U \times V))))=\operatorname{cl}(\operatorname{cbh}(T(U \times V))) .
\end{aligned}
$$

The theorem of Mazur which says that the closed convex hull of a relatively compact subset of a Banach space is compact can also be stated for closed convex balanced hulls. Therefore we have that the image of the ball of radius $\frac{1}{2}$ under $\hat{T}$ is trapped in a compact set, and hence $\hat{T}$ is compact.

If $T_{1}: E_{1} \rightarrow F_{1}$ and $T_{2}: E_{2} \rightarrow F_{2}$ are compact, then so is $T_{1} \hat{\otimes} T_{2}: E_{1} \hat{\otimes} E_{2} \rightarrow$ $F_{1} \hat{\otimes} F_{2}$. This fact follows from the following commutative diagram

$$
\begin{array}{cl}
E_{1} \times E_{2} \stackrel{\phi_{E}}{\longrightarrow} E_{1} & \hat{\otimes} F_{2} \\
T_{1} \times T_{2} \downarrow & \underset{\downarrow}{\downarrow} T_{1} \hat{\otimes} T_{2} \\
F_{1} \times F_{2} \underset{\phi_{F}}{\longrightarrow} F_{1} \otimes F_{2} &
\end{array}
$$

the compactness of $T_{1} \times T_{2}$, and the result above.

\section{BiBLIOGRAPHY}

1. J. Dieudonné, Foundations of modern analysis, Academic Press, New York, 1969.

2. S. Mazur and W. Orlicz, Grundlegende Eigenschaften der polynomischen Operationen, Studia Math. 5 (1934), 50-68.

3. F. Treves, Topological vector spaces, distributions and kernels, Academic Press, New York, 1967. MR 37 \#726.

4. M. M. Vainberg, Variational methods for the study of nonlinear operations, GITTL, Moscow, 1956; English transl., Holden-Day, San Francisco, Calif., 1964. MR 19, 567; MR 31 \#638.

Johns Hopkins University, Baltimore, Maryland 21218 